\title{
Cathodic Polarization Coats Titanium Based Implant Materials with Enamel Matrix Derivate (EMD)
}

\section{Matthias J. Frank ${ }^{1,2}$, Martin S. Walter ${ }^{1,2}$, Marina Rubert ${ }^{3}$, Bernd Thiede ${ }^{4}$, Marta Monjo ${ }^{1,3}$, Janne E. Reseland ${ }^{1}$, Håvard J. Haugen ${ }^{1, *}$ and Ståle Petter Lyngstadaas ${ }^{1}$}

1 Department of Biomaterials, Institute for Clinical Dentistry, University of Oslo, P.O. Box 1109 Blindern, Oslo NO-0317, Norway; E-Mails: m.j.frank@ odont.uio.no (M.J.F.); m.s.walter@odont.uio.no (M.S.W.); marta.monjo@uib.es (M.M.); j.e.reseland@odont.uio.no (J.E.R.); s.p.lyngstadaas@odont.uio.no (S.P.L.)

2 Institute of Medical and Polymer Engineering, Technische Universität München, Boltzmannstrasse 15, Garching 85748, Germany

3 Department of Fundamental Biology and Health Sciences, Research Institute on Health Sciences (IUNICS), University of Balearic Islands, Palma de Mallorca ES-07122, Spain;

E-Mail: marinarubert@gmail.com

4 The Biotechnology Centre of Oslo, University of Oslo, P.O. Box 1125 Blindern, Oslo NO-0317, Norway; E-Mail: bernd.thiede@ biotek.uio.no

* Author to whom correspondence should be addressed; E-Mail: h.j.haugen@ odont.uio.no; Tel.: +47-22-85-21-70; Fax: +47-22-85-23-51.

Received: 29 January 2014; in revised form: 5 March 2014 / Accepted: 10 March 2014 / Published: 14 March 2014

Abstract: The idea of a bioactive surface coating that enhances bone healing and bone growth is a strong focus of on-going research for bone implant materials. Enamel matrix derivate (EMD) is well documented to support bone regeneration and activates growth of mesenchymal tissues. Thus, it is a prime candidate for coating of existing implant surfaces. The aim of this study was to show that cathodic polarization can be used for coating commercially available implant surfaces with an immobilized but functional and bio-available surface layer of EMD. After coating, XPS revealed EMD-related bindings on the surface while SIMS showed incorporation of EMD into the surface. The hydride layer of the original surface could be activated for coating in an integrated one-step process that did not require any pre-treatment of the surface. SEM images showed nano-spheres and nano-rods on coated surfaces that were EMD-related. Moreover, the surface roughness remained unchanged after coating, as it was shown by optical profilometry. The mass 
peaks observed in the matrix-assisted laser desorption/ionization time-of-flight mass spectroscopy (MALDI-TOF MS) analysis confirmed the integrity of EMD after coating. Assessment of the bioavailability suggested that the modified surfaces were active for osteoblast like MC3M3-E1 cells in showing enhanced Coll-1 gene expression and ALP activity.

Keywords: bioactive coating; coating technique; enamel matrix derivate; EMD; titanium; titanium-zirconium; surface modification

\section{Introduction}

Titanium based endosseous dental implants have shown improved clinical performance over recent years [1]. Although the response of bone tissue to an endosseous dental implant is governed by several factors, the implant's surface morphology and surface chemistry largely influence the biological response to an implant [2,3]. Modifications of the surface roughness and chemistry to improve bone healing by machining, sand-blasting, acid-etching, or their combination, are common approaches for modifying the surface chemistry and for altering the surface topography and morphology on a micro- and nano-scale [4-9]. Blasting and etching have been shown to be successful in optimizing surface roughness and enhancing surface reactivity by increasing surface hydride levels for improved clinical performance [10-19]. Reducing environmental carbon contamination has been shown to further enhance the surface energy and wettability of sand-blasted and acid-etched (SBAE) surfaces. Such a reactive surface can be maintained by handling under protective cover gas and storage in saline solution [20,21]. A different approach to improve the surface by etching in hydrofluoric acid has shown elevated hydrogen levels and traces of fluoride on the surface [22,23]. Dental implants with fluoride-doped titanium dioxide have demonstrated enhanced osteoblast differentiation and bone growth [24-26].

Another, but less explored, approach in improving implant surfaces is the biochemical modification of the surface. A commonly used approach is the biomimetic deposition of hydroxyapatite (HA) on metallic surfaces [27]. Surfaces coated with HA have shown improved in vivo performance compared to untreated surfaces [28]. Moreover it has been shown that a HA layer may be used for incorporating biomolecules like proteins or antibiotics [29-32]. A variety of other successful biochemical surface modifications with peptides and extracellular matrix proteins and have shown that biochemically modified surfaces can improve bone healing compared to unmodified titanium surfaces [33,34]. As there is a variety of biomolecules available that promote bone healing, a surface modification by chemically attaching such a biomolecule to the surface of an implant directly offers a potential for enhancing implant performance in bone whilst maintaining the characteristics of the original implant. However, the challenges of making a bioactive coating bioavailable and to maintain its function remain [34].

Lyngstadaas and Ellingsen suggested using a polarization process to attach charged biomolecules to the surface in order to stimulate bone healing [35]. Cathodic polarization in acidic solution creates a hydride layer on titanium or titanium alloys that can be used as an activated surface for attaching 
charged biomolecules [35]. A previous study, which used such a process on smooth, commercially pure titanium showed that cathodic reduction of titanium in acidic solutions was successfully used in order to create thick hydride layers on the surface [36]. Our previous study showed that cathodic polarization on a SBAE surface exhibited a non-linear but cyclic development of the hydride layer [37]. Such a cyclic hydrogen development offers the potential for attaching charged biomolecules to the surface even faster than on commercially pure titanium like we have shown for strontium and doxycycline in our previous studies [38,39].

In search for a candidate biomolecule which could be attached to the surface of an implant by the proposed polarization process, enamel matrix derivate (EMD) appeared to be a promising candidate as it mainly contains amelogenins, which are the major component of the enamel extracellular matrix [40]. Lyngstadaas et al. [41] showed the potential of EMD for use in bone regeneration and implantology. Promising results of EMD supporting periodontal bone regeneration and the angiogenic effect of EMD have been shown in various studies [42-44]. Moreover, major components in EMD have been reported to have bipolar properties, which are a requirement for being used in an electro-coating process [45-47]. Hence, this study chose to use EMD for exploring the feasibility of a bioactive surface coating by means of the aforementioned polarization process.

The aim of this study was to show that a cathodic polarization process can be used for coating EMD onto commercially available dental implant surfaces. The secondary aim of this study was to show that EMD was bio-available and maintained its function after coating.

\section{Results}

\subsection{Surface Chemistry}

Depth profiles acquired by SIMS (Figure 1, Table 1) revealed increased carbon layer thickness, total carbon and total hydrogen content for TiZr EMD and Ti EMD compared to the respective SBAE surfaces. In detail, total carbon was increased 7.6-fold and total hydrogen was increased 2.1-fold for TiZr EMD compared to TiZr SBAE, while Ti EMD showed a 22-fold increase in total carbon and a 3.9-fold increase in hydrogen levels when compared to Ti SBAE. A similar trend was observed for the maximum intensity of hydrogen and carbon.

XPS analysis revealed that pure EMD consisted mainly of carbon (C 1s) at $60.91 \%$ (Table 2), whereas $52.72 \%$ of this carbon was present as a carbon single bond $(\mathrm{C}-\mathrm{C}), 20.81 \%$ as a double bond $(\mathrm{C}=\mathrm{C})$, and $26.47 \%$ as a single carbon-oxygen bond $(\mathrm{C}-\mathrm{O})$ (Table 3$)$. Oxygen $(\mathrm{O} 1 \mathrm{~s})$ was the second largest component of pure EMD at $24.69 \%$, whereas $80.62 \%$ of this oxygen was bound as organic oxygen and $19.38 \%$ as a carbon-oxygen single bond (not shown in the table). In addition to carbon and oxygen, pure EMD also showed $13.9 \%$ of nitrogen (N 1s) and traces of silica (Si 2p) and sulfur (S 2p).

EMD-coated surfaces of both materials showed a shift in the binding state of the carbon on the surface towards a distribution of $\mathrm{C}-\mathrm{C}, \mathrm{C}=\mathrm{C}$, and $\mathrm{C}-\mathrm{O} / \mathrm{C}-\mathrm{N}$ bonds that was comparable to the distribution observed for pure EMD. TiZr EMD and Ti EMD showed an increase in $\mathrm{C}=\mathrm{C}$ bonds of circa 10 pp compared to the SBAE surface (Table 3). Moreover, the specific bonds of oxygen showed over 70 pp more organically bond oxygen for EMD-coated samples than for SBAE samples. While 
TiZr EMD and Ti EMD also showed Si and N, only TiZr EMD had S on the surface as had been observed for pure EMD.

Figure 1. SIMS depth profiles of the ${ }^{12} \mathrm{C}(\mathbf{A}) ;{ }^{1} \mathrm{H}(\mathbf{B})$ and ${ }^{18} \mathrm{O}(\mathbf{C})$ isotopes.
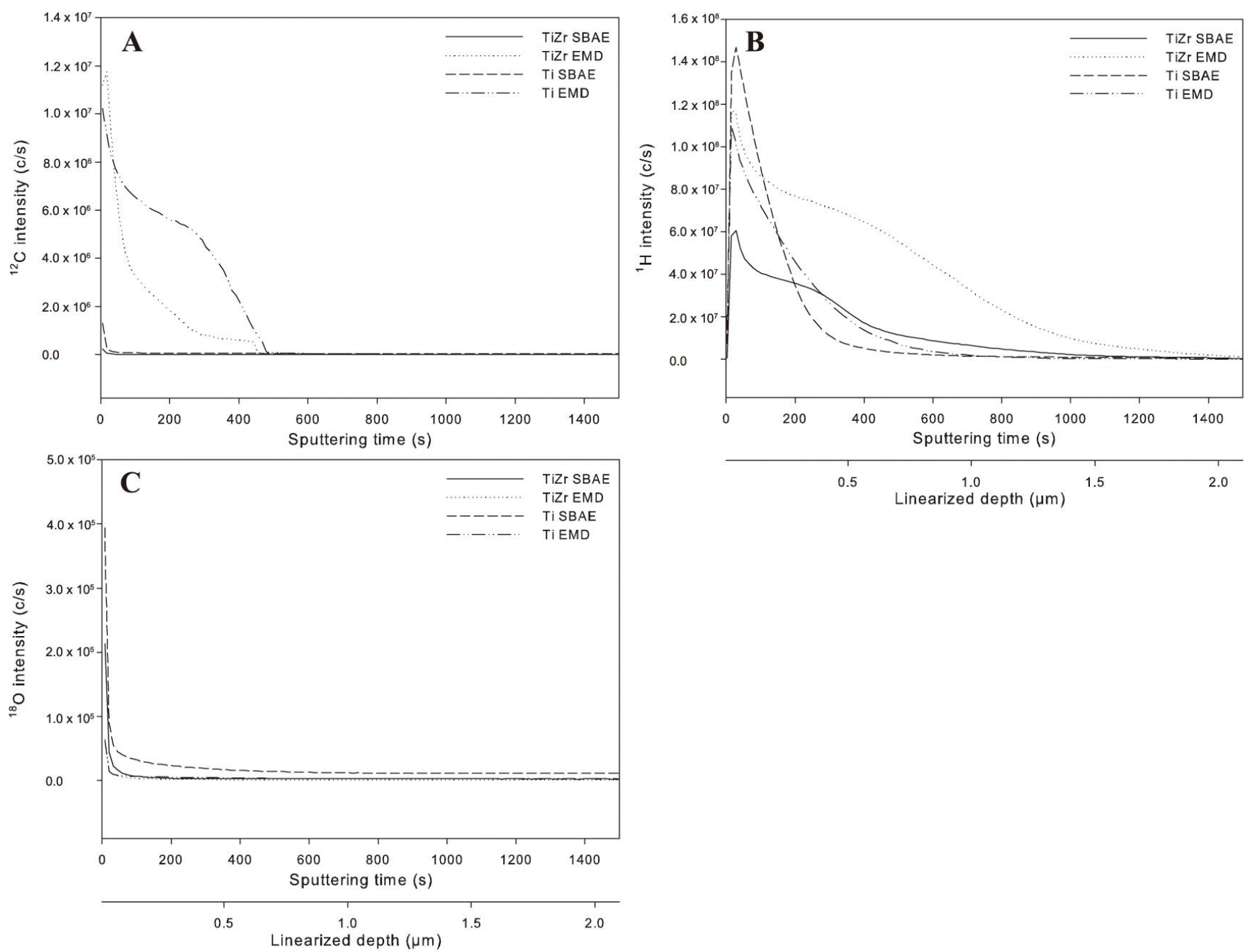

Table 1. SIMS depth profile analysis parameters for the ${ }^{1} \mathrm{H}$ and ${ }^{12} \mathrm{C}$ isotope.

\begin{tabular}{ccccc}
\hline Isotope & Sample & Total amount $(\mathbf{c})$ & Maximum intensity $(\mathbf{c} / \mathbf{s})$ & Layer thickness $(\boldsymbol{\mu m})$ \\
\hline${ }^{1} \mathrm{H}$ & TiZr SBAE & $2.65 \times 10^{7}$ & $6.05 \times 10^{7}$ & 2.26 \\
${ }^{1} \mathrm{H}$ & TiZr EMD & $5.67 \times 10^{7}$ & $1.28 \times 10^{8}$ & 2.92 \\
${ }^{1} \mathrm{H}$ & Ti SBAE & $5.44 \times 10^{6}$ & $1.47 \times 10^{8}$ & 1.1 \\
${ }^{1} \mathrm{H}$ & Ti EMD & $2.10 \times 10^{7}$ & $1.07 \times 10^{8}$ & 1.3 \\
${ }^{12} \mathrm{C}$ & TiZr SBAE & $3.61 \times 10^{4}$ & $2.42 \times 10^{5}$ & 0.06 \\
${ }^{12} \mathrm{C}$ & TiZr EMD & $2.76 \times 10^{5}$ & $5.94 \times 10^{6}$ & 0.75 \\
${ }^{12} \mathrm{C}$ & Ti SBAE & $1.53 \times 10^{5}$ & $1.29 \times 10^{6}$ & 0.03 \\
${ }^{12} \mathrm{C}$ & Ti EMD & $3.37 \times 10^{6}$ & $1.02 \times 10^{7}$ & 1.16 \\
\hline
\end{tabular}


Table 2. XPS surface elements distribution.

\begin{tabular}{cccccc}
\hline \multirow{2}{*}{ Element } & $\begin{array}{c}\text { Pure EMD } \\
(\boldsymbol{\%})\end{array}$ & $\begin{array}{c}\text { TiZr SBAE } \\
(\boldsymbol{\%})\end{array}$ & $\begin{array}{c}\text { TiZr EMD } \\
(\boldsymbol{\%})\end{array}$ & $\begin{array}{c}\text { Ti SBAE } \\
(\boldsymbol{\%})\end{array}$ & $\begin{array}{c}\text { Ti EMD } \\
(\boldsymbol{\%})\end{array}$ \\
\hline O 1s & 24.69 & 53.79 & 19.58 & 54.24 & 23.64 \\
C 1s & 60.91 & 20.74 & 62.55 & 22.74 & 56.66 \\
F 1s & - & 0.78 & - & 1.29 & 0.87 \\
Ti 2p & - & 19.45 & 1.91 & 21.73 & 2.91 \\
N 1s & 13.90 & - & 12.63 & - & 8.64 \\
Cl 2p & - & 1.55 & 0.59 & - & 0.69 \\
Na KLL & - & 0.78 & 1.27 & - & 5.76 \\
Si 2p & 0.15 & - & 0.92 & - & 0.83 \\
S 2p & 0.36 & - & 0.17 & - & - \\
Zr 3d & - & 2.90 & 0.37 & - & - \\
\hline
\end{tabular}

Table 3. XPS specific bindings of the surface oxygen and carbon.

\begin{tabular}{|c|c|c|c|c|c|c|c|c|c|c|c|}
\hline \multirow[b]{2}{*}{ Element } & \multirow[b]{2}{*}{ Assignment } & \multicolumn{2}{|c|}{ Pure EMD } & \multicolumn{2}{|c|}{ TiZr SBAE } & \multicolumn{2}{|c|}{ TiZr EMD } & \multicolumn{2}{|c|}{ Ti SBAE } & \multicolumn{2}{|c|}{ Ti EMD } \\
\hline & & $\begin{array}{c}\text { Position } \\
(\mathrm{eV})\end{array}$ & $\begin{array}{l}\text { Conc. } \\
\text { (at\%) }\end{array}$ & $\begin{array}{c}\text { Position } \\
(\mathrm{eV})\end{array}$ & $\begin{array}{l}\text { Conc. } \\
\text { (at\%) }\end{array}$ & $\begin{array}{c}\text { Position } \\
(\mathrm{eV})\end{array}$ & $\begin{array}{l}\text { Conc. } \\
\text { (at\%) }\end{array}$ & $\begin{array}{c}\text { Position } \\
(\mathrm{eV})\end{array}$ & $\begin{array}{l}\text { Conc. } \\
\text { (at\%) }\end{array}$ & $\begin{array}{c}\text { Position } \\
(\mathrm{eV})\end{array}$ & $\begin{array}{l}\text { Conc. } \\
\text { (at\%) }\end{array}$ \\
\hline $\mathrm{O} 1 \mathrm{~s}$ & & & & & & & & & & & \\
\hline $\mathrm{O} 1 \mathrm{~s}$ & & - & - & 529 & .65 & 529 & & 525 & & 529.09 & 10.96 \\
\hline $\mathrm{C} 1 \mathrm{~s}$ & $\mathrm{C}-\mathrm{C}, \mathrm{CH}_{x}$ & 284.75 & 52.72 & 284.71 & 56.95 & 284.69 & 47.94 & 284.69 & 59.17 & 284.73 & 51.31 \\
\hline & & 287.92 & & & & 287.8 & & 288.81 & & 288.05 & 25.65 \\
\hline $\mathrm{C} 1 \mathrm{~s}$ & $\mathrm{C}-\mathrm{O}, \mathrm{C}-\mathrm{N}$ & 286.05 & 26.47 & 286.10 & 32.70 & 285.95 & 31.12 & 286.10 & 26.44 & 285.93 & 23.04 \\
\hline
\end{tabular}

\subsection{Surface Morphology}

Comparison of TiZr EMD against TiZr SBAE by SEM showed changes to the topography for the micro- and nano-topography. Although the micro-topography of SBAE and the coated samples (Figure 2A,C) revealed similar nano-nodules and small spherical structures on the surface, only the EMD coated sample presented additional larger spherical structures that were not visible for the SBAE sample. At larger magnifications (Figure 2B,D) these larger spherical structures, ranging from $70 \mathrm{~nm}$ to $650 \mathrm{~nm}$ in diameter, appeared to be attached to the surface and showed interconnections to each other. Although, these spherical structures appeared to attach preferably to the peaks of the surface, they were visible on the side faces of the surface peaks as well. The polarization process did not show any other changes to the surface's topography as the edges and peaks appeared to have the same morphology after polarization as they had prior to polarization. Ti SBAE revealed a similar micro-topography of the surface as it was observed for TiZr SBAE (Figure 2A,E), however the surfaces differed at the nano-level (Figure 2B,F). While TiZr SBAE exhibited nano-nodules and spherical structures, none of the two could be observed for Ti SBAE. By contrast, Ti EMD (Figure 2G) showed spherical microstructures that were of the same size as the spherical structures observed for TiZr EMD at larger magnification (Figure 2H). The structures appeared to preferably cover the peaks and edges of EMD-coated surfaces. Apart from the spherical structures described, there were no other changes of the surface topography observed. 
Figure 2. SEM images of (A,B) TiZr SBAE; (C,D) TiZr EMD; (E,F) Ti SBAE and $(\mathbf{G}, \mathbf{H})$ Ti EMD.
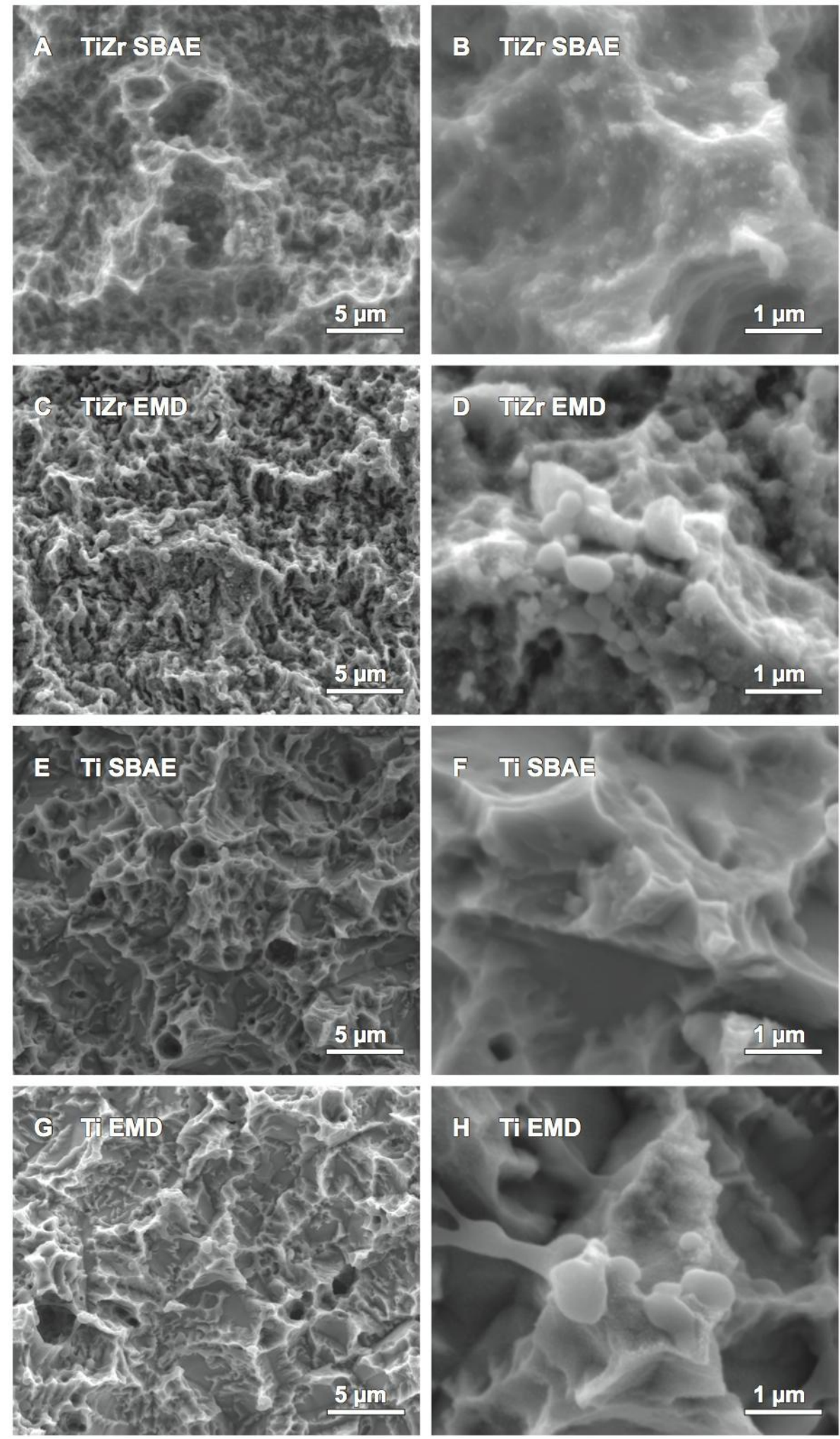

Assessment of the surface micro-topography by optical imaging profilometry only revealed significant changes $(p=0.013)$ against the original SBAE surface for the $S_{a}$ of TiZr EMD (Table 4). The $S_{a}$ was $0.08 \mu \mathrm{m}$ less rough for TiZr EMD than for TiZr SBAE. There appeared to be a general 
difference between the two materials, regardless of the surface treatment, as $S_{\mathrm{a}}, S_{\mathrm{sk}}$ and $S_{\mathrm{ci}}$ revealed higher values for TiZr, and $S_{\mathrm{ku}}$ and $S_{\mathrm{dr}}$ revealed higher values for Ti.

Table 4. Mean surface topography parameters with standard deviation assessed by optical imaging profilometry. Only TiZr EMD showed a significant difference $\left.*^{*} p<0.05\right)$ for $\mathrm{S}_{\mathrm{a}}$ compared to TiZr SBAE.

\begin{tabular}{cccccc}
\hline Sample & $\mathbf{S}_{\mathbf{a}}(\boldsymbol{\mu} \mathbf{m})$ & $\mathbf{S}_{\mathbf{s k}}$ & $\mathbf{S}_{\mathbf{k u}}$ & $\mathbf{S}_{\mathbf{c i}}$ & $\mathbf{S}_{\mathbf{d r}}(\boldsymbol{\%})$ \\
\hline TiZr SBAE & $2.074 \pm 0.08$ & $-0.142 \pm 0.07$ & $2.920 \pm 0.14$ & $1.530 \pm 0.03$ & $58.19 \pm 2.14$ \\
TiZr EMD & $1.904 \pm 0.06 *$ & $-0.146 \pm 0.09$ & $2.939 \pm 0.05$ & $1.528 \pm 0.03$ & $58.78 \pm 5.06$ \\
Ti SBAE & $1.861 \pm 0.12$ & $-0.195 \pm 0.35$ & $3.800 \pm 1.07$ & $1.433 \pm 0.09$ & $70.51 \pm 4.94$ \\
Ti EMD & $1.816 \pm 0.04$ & $-0.359 \pm 0.13$ & $3.641 \pm 0.81$ & $1.448 \pm 0.05$ & $72.51 \pm 3.21$ \\
\hline
\end{tabular}

\subsection{Assessment of EMD's Integrity}

The matrix-assisted laser desorption/ionization time-of-flight mass spectroscopy (MALDI-TOF MS) spectrum of pure EMD in $0.1 \%$ acetic acid revealed a very intense peak in the $5 \mathrm{kDa}$ region and a less intense peak at $16.9 \mathrm{kDa}$ (Figure 3A). Matrix-assisted laser desorption/ionization in source decay (MALDI-ISD) of pure EMD revealed fragments with the sequence YEVLTPLKWYQNM (Figure 4). TiZr EMD showed a major peak at 5179 Da ( 620 a.u.) (Figure 3B). This peak was surrounded by peaks with a mass difference of $16 \mathrm{Da}$. Ti EMD showed its major peak at $5180 \mathrm{Da}$ ( 330 a.u.) (Figure 3C). As has been observed for TiZr EMD, this peak was also surrounded by peaks with a mass difference of $16 \mathrm{Da}$ and $17 \mathrm{Da}$. Moreover, Ti EMD also revealed a peak at $16.9 \mathrm{kDa}$.

Figure 3. MALDI spectra of (A) pure EMD; (B) TiZr EMD and (C) Ti EMD. All graphs include an enlarged version of the region between 5000 and $5400 \mathrm{~m} / \mathrm{z}$.

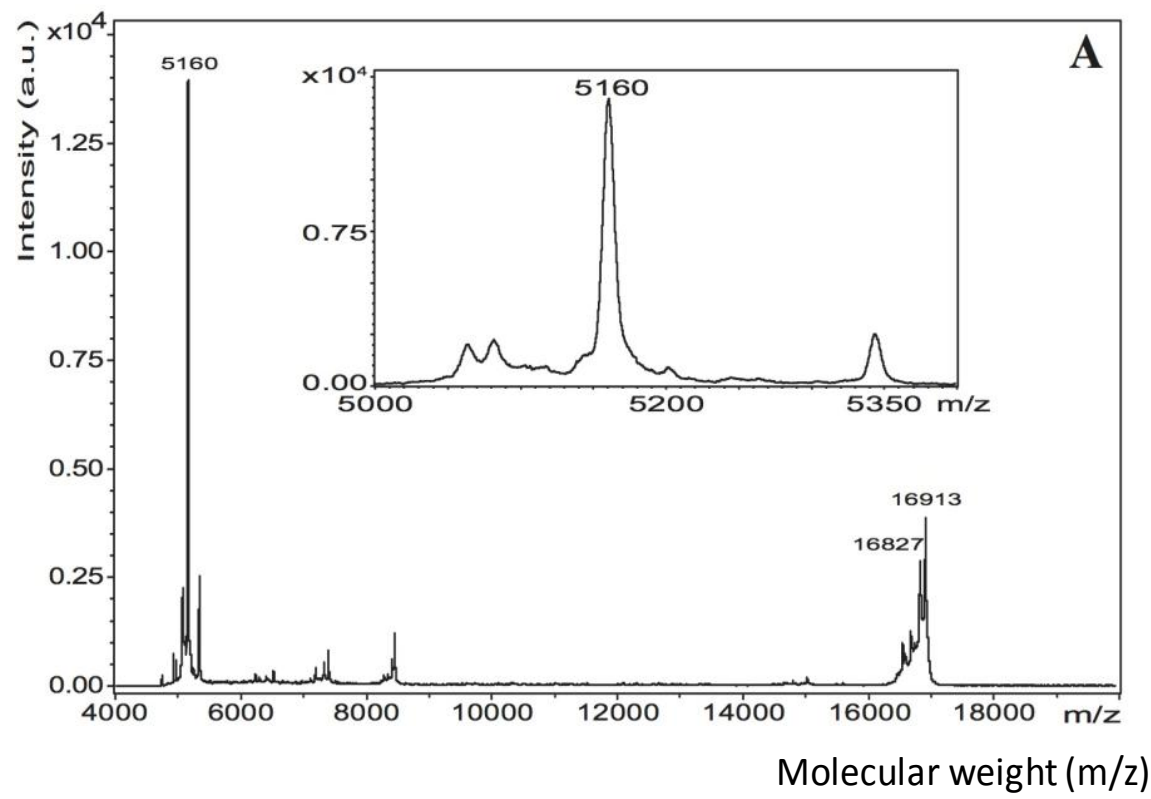


Figure 3. Cont.
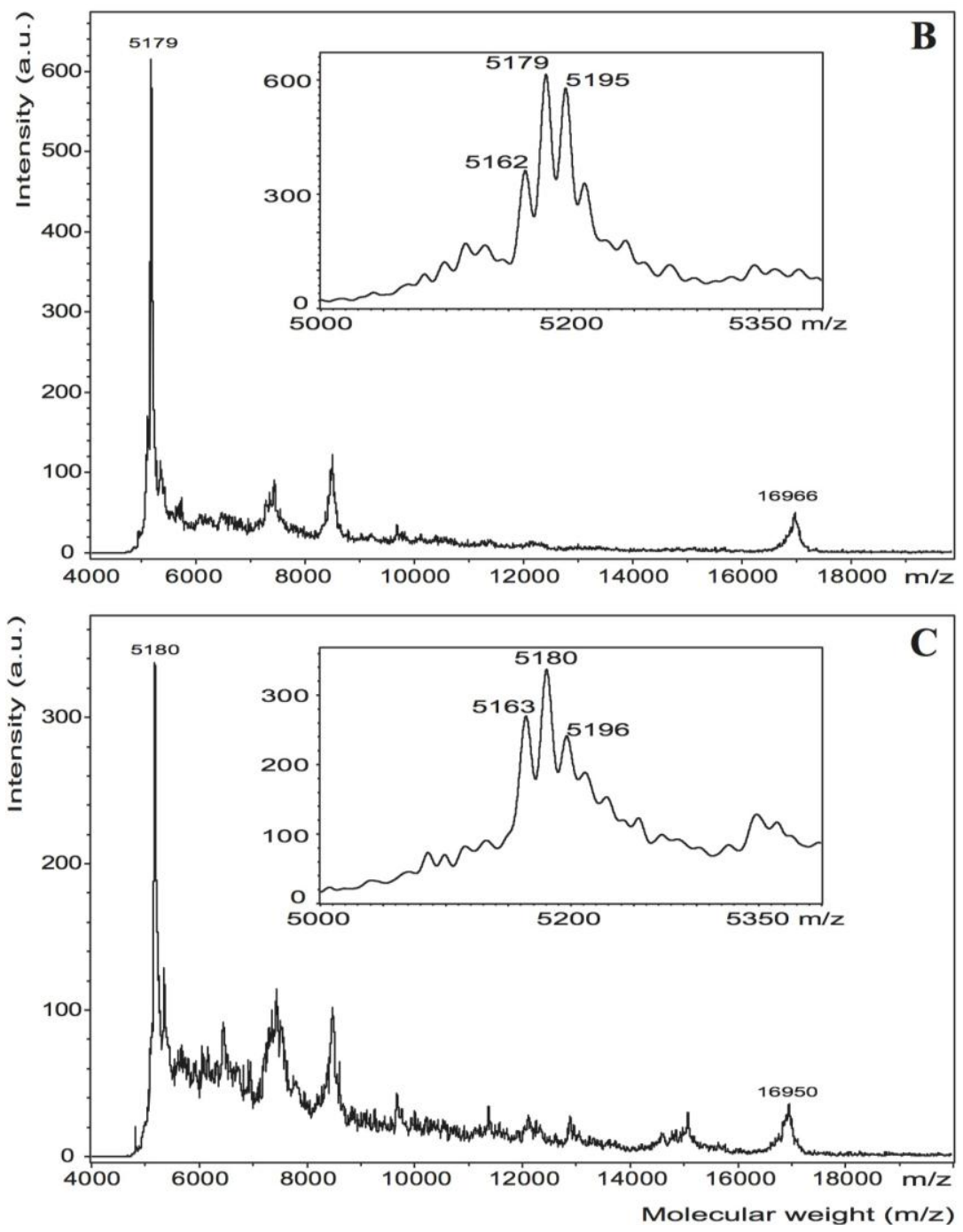

Figure 4. MALDI-ISD of EMD revealed fragments with the sequence YEVLTPLKWYQNM corresponding to the amino acid stretch 33-45.

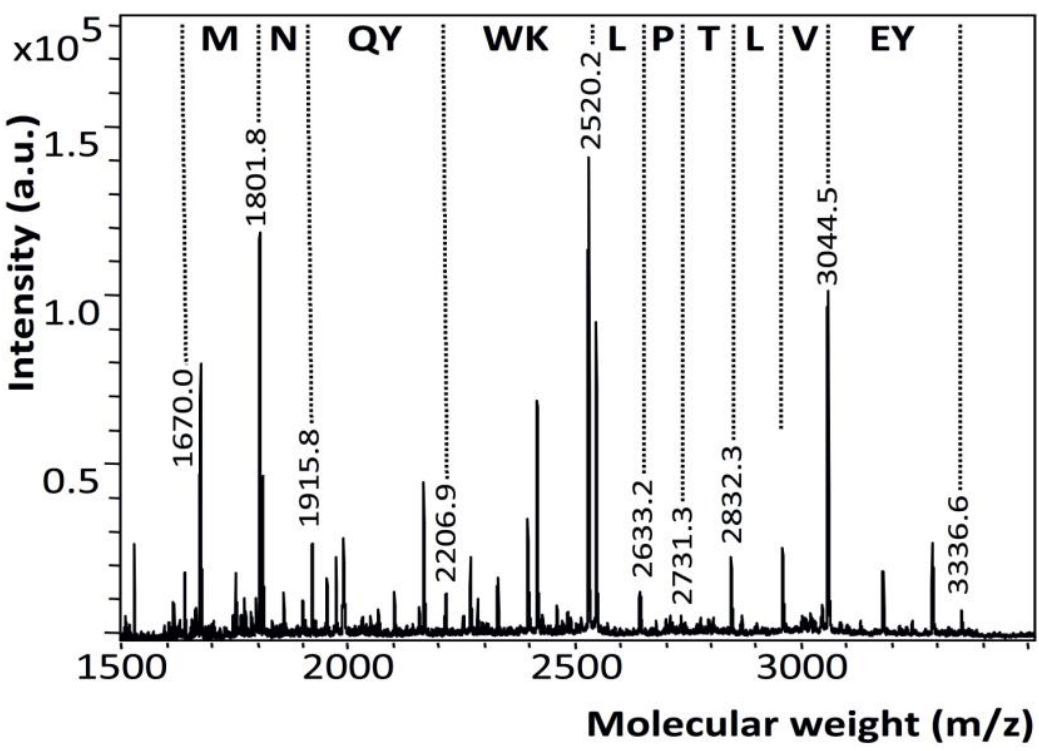




\subsection{EMD Bioavailability Assessment}

Electro-coated TiZr EMD displayed statistically significant differences $(p<0.05)$ for the expression of Coll-1 mRNA levels (Figure 5A) and alkaline phosphatase (ALP) activity (Figure 5B) compared to TiZr SBAE. Like for TiZr EMD, ALP activity was significantly different for Ti EMD compared to Ti SBAE. By contrast, the expression of Coll-1 mRNA levels of Ti EMD was not significantly higher compared to Ti SBAE, although higher gene expression was observed. The pol groups did not expose any significant differences in gene expression compared to TiZr SBAE.

Figure 5. Coll-1 relative mRNA levels (A) and ALP activity for polarized only and EMD coated groups were displayed as box plots of the median values (Q2) with 5, 25 (Q1), $75(\mathrm{Q} 3)$ and 95 percentiles $(n=6)$. Student's t-test revealed significant $(* p \leq 0.05)$ differences in Coll-I relative mRNA levels between TiZr EMD and TiZr SBAE. ALP activity revealed significant differences between TiZr EMD and TiZr SBAE and between Ti EMD and Ti SBAE.

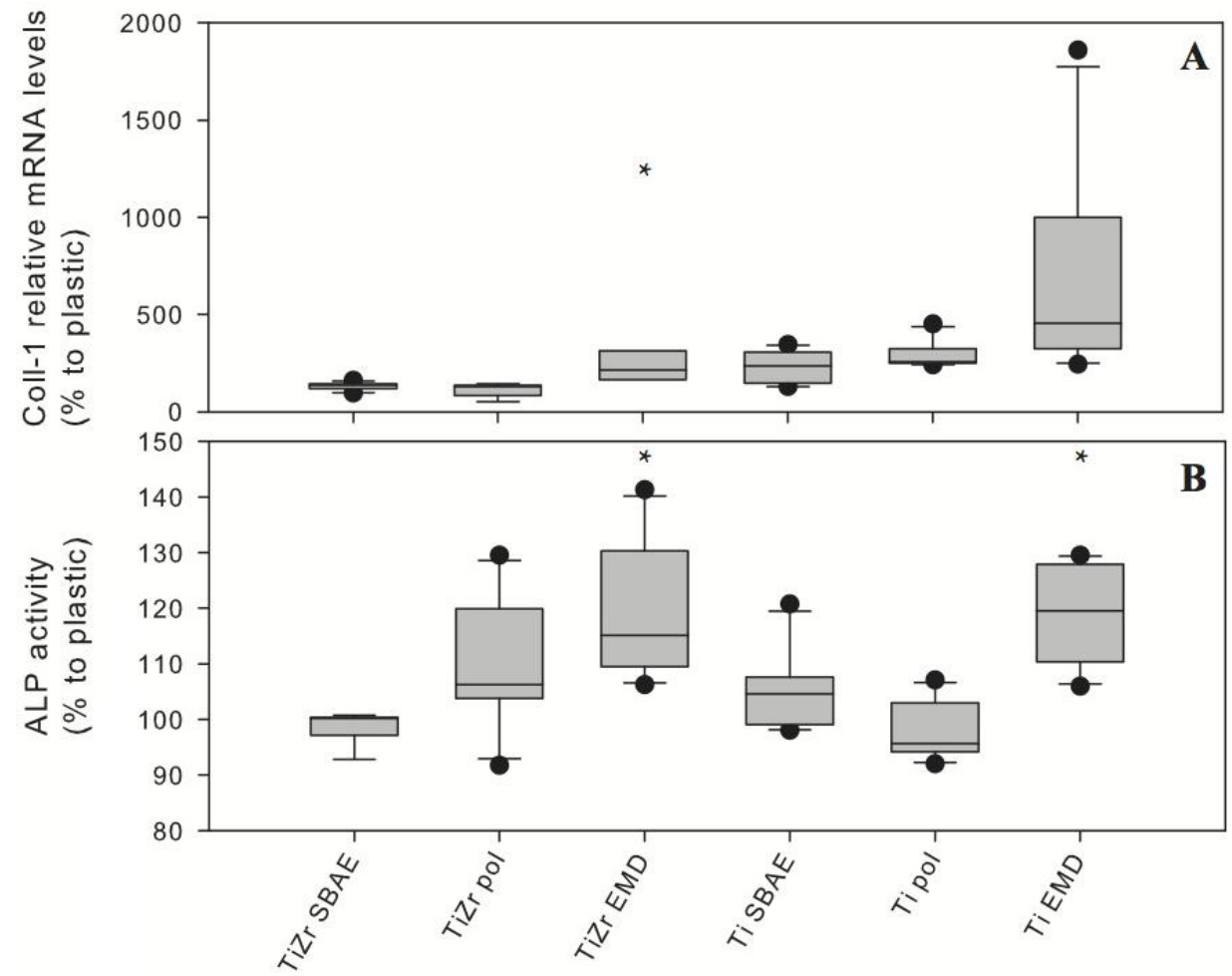

\section{Discussion}

\subsection{EMD Bioavailability Assessment of the Coated Surfaces}

TiZr EMD exhibited earlier proliferation than TiZr SBAE, concluding from the significantly increased expression of Coll-1 and ALP activity based on the temporal expression presented by Quarles et al. [48] and Monjo et al. [49]. Likewise, Ti EMD tended towards earlier cell proliferation than Ti SBAE, based on significantly higher ALP activity and a trend towards increased expression of Coll-1, although this difference was not statistically significant. As TiZr pol and Ti pol did not show any significant differences to the performance of the respective SBAE group, the enhanced cell 
proliferation was linked to the EMD-coating of the surface and confirmed that EMD was not only bio-available but also maintained its function. The findings presented for EMD-coated surfaces agreed with the results reported by Reseland et al. [50], who also showed increased Coll-1 levels and ALP activity for primary human osteoblasts incubated with EMD. A different study by Rubert et al. [51] has also shown increased Coll-1 mRNA levels after 14 days for MC3T3-E1 cells incubated with EMD. A recent in vitro study using rat calvarial osteoblasts by Miron et al. [43] assessed a titanium SLA ${ }^{\circledR}$ surface (Straumann, Basel, Switzerland), similar to the Ti SBAE surface used in this study, that was coated with EMD by a dipping procedure. Their study showed enhanced cell proliferation and cell differentiation for EMD-modified Ti SLA ${ }^{\circledR}$. Miron et al. [43] concluded that EMD accelerated differentiation by promoting mature phenotypes earlier than $\mathrm{Ti}$ alone.

\subsection{Confirmation of EMD's Integrity after Coating}

As EMD consists of a multitude of components of different size and mass, MALDI was used to analyze the individual fractions of an EMD-coated surface in order to confirm the integrity of the coating. The MALDI spectra of TiZr EMD and Ti EMD showed peaks in the region of $5 \mathrm{kDa}$ and $16 \mathrm{kDa}$ comparable to the peaks found for pure EMD. The minor differences in peak mass observed were not unusual when comparing it to the results of other authors [52,53]. Mumulidu et al. [52] reported a similar range for the $5 \mathrm{kDa}$ peak of EMD when analyzing this peak specifically. They concluded that the mass variation observed for this peak might be a result of the small amount of the sample itself [52]. Moreover, this study showed the major peak in the $5 \mathrm{kDa}$ region to be surrounded by peaks with a mass difference of $+16 \mathrm{Da}$ that were corresponding to oxidation. The peak found at 16.9 kDa for pure EMD was also observed for TiZr EMD and Ti EMD. Riksen et al. [54] showed a peak at 16.6 $\mathrm{kDa}$ for two different amelogenin fractions separated from EMD by size-exclusion high-performance liquid chromatography. As Riksen et al. [54] used 0.05\% acetic acid for dissolving EMD, the $\mathrm{pH}$ should have been slightly higher than for the $0.1 \%$ acetic acid solution used in this study. Cohen et al. [55] described a pH dependency of the mass peak of a MALDI measurement when using a HCCA matrix like it was used in this study. While pure EMD was dissolved in $0.1 \%$ acetic acid at pH 3.1, the ACN+TFA solution used for detaching EMD from the coated coins had a pH of 1.8. It was believed that the results obtained by Riksen et al. [54] were measured at a $\mathrm{pH}$ that was different to the $\mathrm{pH}$ used for the samples of this study. Hence, an influence of the different $\mathrm{pH}$ values of the single samples could not be excluded. Even though EMD consists of a multitude of components of different size and weight, a successful coating with EMD was supported by MALDI as the fractions found on EMD coated surfaces largely corresponded with the fractions observed for pure EMD.

The peaks observed in the MALDI spectrum of pure EMD in the $5 \mathrm{kDa}$ and $16 \mathrm{kDa}$ region concurred with the peaks that have previously been reported in the literature [40,52-54]. Moreover, sequencing of pure EMD MALDI-ISD revealed fragments with the sequence YEVLTPLKWYQNM that were corresponding to the amino acid stretch 33-45. Considering the detected molecular mass at $5158 \mathrm{Da}$ and the determined sequence, this molecule corresponded to amelogenin, which is the major component of EMD, sequence stretch 17-59 without the signal peptide and with an additional mass of $80 \mathrm{Da}$ within sequence stretch 17-32 [40,53]. This mass difference indicated towards a phosphorylation. Notably, serine-32 has been identified to be phosphorylated in bovine amelogenin [53]. 


\subsection{Chemical Confirmation of an Effective Surface Coating with EMD}

The increased carbon layer thickness and total carbon content for TiZr EMD and Ti EMD observed by SIMS suggested an incorporation of EMD into the surface. Detailed analysis of the coated surfaces by XPS showed a shift in the carbon binding state on the outer surface towards a binding state distribution of $\mathrm{C}-\mathrm{C}, \mathrm{C}=\mathrm{C}$, and $\mathrm{C}-\mathrm{O} / \mathrm{C}-\mathrm{N}$ bonds that was comparable to the distribution observed for pure EMD. Moreover, surface oxygen was significantly decreased for EMD-coated samples while the oxygen binding state was shifted from Ti-bond oxygen towards organically bound oxygen, which correlated with the binding state of pure EMD, which has about $80 \%$ organically bound oxygen. In addition, EMD related nitrogen, silicon, and sulfur that have not been detected on the initial SBAE surfaces could be observed for EMD-coated surfaces. The decrease in $\mathrm{Ti}$ and $\mathrm{Zr}$ levels on the surface for EMD-coated samples indicated that the surface was masked by the EMD-coating like it has been described by Morra et al. [56] for a different surface coating.

The presence of fluoride on SBAE and EMD surfaces was not intended and was believed to have derived from handling of the samples with PTFE covers during blasting, etching and polarization. The presence of $\mathrm{Na}$ and $\mathrm{Cl}$ was believed to be a result of the storage of the SBAE samples in saline solution. Even though the SBAE samples were washed in reverse osmosis deionized water in an ultrasonic bath, for $5 \mathrm{~min}$, prior to examination or polarization, an adhesion of $\mathrm{NaCl}$ to the respective surface remained possible and was most likely the source of these trace elements. The results observed for the SBAE surfaces were in accordance with previous studies on similar surfaces [56,57].

The hydride layer created by this process was proposed to be the linking element between the metal surface and the biomolecule. Therefore, the hydrogen depth profile was of particular interest. The results of this study showed increased hydrogen maximum hydrogen intensity, total hydrogen content, and layer thickness of the EMD-coated samples when compared to the corresponding SBAE samples. This was in agreement with the coating mechanism that has been suggested by Lyngstadaas and Ellingsen [35].

\subsection{Visual Confirmation of Effective Surface Coating with EMD}

The results of the SEM analysis showed large interconnected spherical structures for TiZr EMD and Ti EMD that were not present on the respective SBAE surface. Interestingly, the structures observed for EMD-coated samples were different to the structures observed in our previous study that used the same polarization process without any biomolecules on the same SBAE materials [37]. While the structures observed for polarized SBAE surfaces in our previous study were shaped differently, they also seemed to have grown from inside the material towards the outer surface. By contrast, the spheres observed for EMD-coated samples in this study appeared to have been attached on top of the material from the surroundings. The masking effect of titanium and zirconium respectively previously described supported this observation. Such an attachment would be in concordance with the attachment of EMD to the surface as it was suggested. Moreover, Gestrelius et al. [58,59] showed EMD precipitated from aqueous solution to form spheres or short rods that were comparable to the structures observed for EMD-coated samples in this study. Thus, the spheres and short rods seen on the surface of EMD coated samples in the SEM images of this study were interpreted to be EMD. 
Analysis of the surface roughness parameters by optical imaging profilometry did not reveal significant changes after EMD-coating except for the $S_{a}$ of TiZr EMD. Even though the change was statistically significant, the actual $S_{a}$ of TiZr EMD was midway between the $S_{a}$ of TiZr SBAE and Ti SBAE. The observed trend towards generally different surfaces for TiZr and Ti has been reported in previous studies [10,57]. It has been shown in in vivo studies that TiZr SBAE performed equally well if not better than Ti SBAE [60-62]. Moreover, $S_{a}$ values between 1.16 and $3 \mu \mathrm{m}$ have been shown to optimal for titanium endosseous dental implants with a surface comparable to the SBAE surface [63-65]. Hence, the surface topography created during EMD-coating should not have a negative effect on the performance of the surface but an effect may only be expected from the coating itself.

\section{Experimental Section}

\subsection{Samples}

This study used coin-shaped samples made of grade IV commercially pure titanium (Ti) and a titanium-zirconium alloy (TiZr) containing $13 \%$ to $17 \%$ zirconium with a grit-blasted and acid-etched (SBAE) surface with a diameter of $4.39 \mathrm{~mm}$ and a height of $2 \mathrm{~mm}$. Detailed information about the samples have been described in our previous study [10] and in other studies [13,20]. The setup used for cathodic polarization was the same as it has been described in our previous study [37]. Polarization was performed in $200 \mathrm{~mL}$ of a $2 \mathrm{M}$ buffer solution mixed of acetic acid and sodium acetate at $\mathrm{pH} 5$. $20 \mathrm{mg}$ of dry stored EMD (Institut Straumann AG, Basel, Switzerland) were dissolved in $2 \mathrm{~mL}$ of $0.1 \%$ acetic acid at $4{ }^{\circ} \mathrm{C}$ and added to the buffer for final EMD-concentration of $0.01 \mathrm{mg} / \mathrm{mL}$. The buffer was kept at $21{ }^{\circ} \mathrm{C}$ over the course of the whole process. After processing, all samples were air-dried in a laminar flow cabin for $30 \mathrm{~min}$ and packed in Eppendorf tubes. The coating of TiZr SBAE with EMD (TiZr EMD) was done for $60 \mathrm{~min}$ while the output current was set to $0.49 \mathrm{~mA} / \mathrm{cm}^{2}$. Ti SBAE was coated with EMD (Ti EMD) for $60 \mathrm{~min}$ at a current density of $1.65 \mathrm{~mA} / \mathrm{cm}^{2}$. A control group that was cathodically polarized without addition of EMD to the buffer (TiZr pol; Ti pol) was added to the cell study to assess the effect of the polarization independently of EMD coating. The parameters were chosen for the particular materials based on the results of our previous study that showed a promising development of the hydride levels for attaching charged biomolecules for those settings [37].

\subsection{Chemical Characterization}

TiZr SBAE samples and EMD-coated samples were analyzed by SIMS in order to demonstrate EMD incorporation into the surface by assessment of the ${ }^{12} \mathrm{C}$ isotope's depth profile, as EMD consists to a large part of carbon. Lamolle et al. [9] used this method to show the incorporation of fluorine into the surface of titanium after etching in hydrofluoric acid. Furthermore, depth profiles of the ${ }^{1} \mathrm{H}$ and ${ }^{18} \mathrm{O}$ isotopes were obtained. Analysis was performed on an IMS 7f (Cameca, Paris, France) magnetic sector SIMS using the same parameters that have been described in our previous study [10].

Analysis of the surface by XPS was used to detect changes in the surface chemistry and binding states after polarization with EMD. The XPS analysis was carried out on an Axis Ultra ${ }^{\text {DLD }}$ XP spectrometer (Kratos Analytical Limited, Manchester, UK). Detail spectra were recorded for O 1s and 
$\mathrm{C} 1 \mathrm{~s}$. The energy shift due to surface charging was below $1 \mathrm{eV}$ based on the $\mathrm{C} 1 \mathrm{~s}$ peak position relative to the established BEs, therefore the experiment was performed without charge compensation. All other settings of the instrument were the same that have been described in our previous study [10]. Peaks were interpreted according to Moulder and Chastain [66], and Cai et al. [67].

\subsection{Surface Morphology}

All SEM images in this study were taken on a Quanta 200 FEG (FEI Hillsboro, OR, USA) field-emission SEM. Its Schottky field emission gun (FEG) allowed high spatial resolution. All samples were sputtered with platinum for one minute prior to imaging and mounted on the sample holder with conductive carbon tape.

A PL 2300 (Sensofar-Tech S.L., Terrassa, Spain) blue light laser profilometer and interferometer using a 50x EPI (Nikon, Tokyo, Japan) confocal objective was used for assessing the surface topography. The following surface parameters were analyzed: average roughness $\left(S_{a}\right)$, skewness of the height distribution $\left(\mathrm{S}_{\mathrm{sk}}\right)$, kurtosis of the height distribution $\left(\mathrm{S}_{\mathrm{ku}}\right)$, core fluid retention index $\left(\mathrm{S}_{\mathrm{ci}}\right)$, and developed interfacial area ratio $\left(\mathrm{S}_{\mathrm{dr}}\right)$.

\subsection{Analysis of the Biomolecules}

As EMD is almost insoluble at physiological $\mathrm{pH}$ and temperature, it was detached from the surface in a solution containing $40 \%$ acetonitrile $(\mathrm{ACN})$ and $0.3 \%$ trifluoro acetic acid (TFA) at pH 2 and at $8{ }^{\circ} \mathrm{C}$ [40]. Samples were submerged in $1 \mathrm{~mL}$ of ACN+TFA and placed on a shaker for $24 \mathrm{~h}$. Pure EMD was dissolved in acetic acid as described earlier. All samples were cleaned using a C4-ZipTip to remove impurities that may have created background noise during MALDI-TOF MS. $0.5 \mathrm{~mL}$ of $20 \mathrm{mg} / \mathrm{mL} \alpha$-Cyano-4-hydroxycinnamic acid (HCCA) matrix in 0.3\% TFA/acetonitrile (2:1) was added to each sample before spotting it onto a stainless steel MALDI plate. MALDI-TOF MS was performed on an ULTRAFLEX II MALDI-TOF/TOF (Bruker Daltonics, Bremen, Germany). Basic settings of the instrument for the spectra were as follows: ion source 1: $25 \mathrm{kV}$; ion source 2: $23.5 \mathrm{kV}$; lens: $6.5 \mathrm{kV}$; deflection mass $4000 \mathrm{Da}$, polarity positive. Basic setting for MALDI-in source decay (MALDI-ISD) were as follows: ion source 1: $25 \mathrm{kV}$; ion source 2: $21.85 \mathrm{kV}$; lens: $9.7 \mathrm{kV}$; reflector: $26.3 \mathrm{kV}$; reflector 2: $13.85 \mathrm{kV}$; deflector mode, polarity positive. FlexControl 3.0 (Bruker Daltonics, Bremen, Germany) was utilized for data acquisition and FlexAnalysis 2.4 (Bruker Daltonics, Bremen, Germany) for further analysis.

\subsection{Bio-Availability Study}

An in vitro cell study was performed to evaluate the bioavailability of the biomolecule on the coated surfaces by comparing the EMD-polarized samples against the respective SBAE surface. All groups had a group size of six samples per group. A group that was only polarized without any EMD in the buffer was used as an additional control group. The murine osteoblastic cell line MC3T3-E1 was obtained from the German Collection of Microorganisms and Cell Cultures (DSMZ, Braunschweig, Germany). All experiments were performed in the same passage of the MC3T3-E1 cells (passage 16). The same number of cells was cultured in parallel on plastic for all experiments and the results were 
presented as relative to the expression of the plastic control group in percent. The detailed steps for cell culturing, used housekeeping genes and analytical methods for RNA isolation, real time polymerase chain reaction analysis and determination of ALP activity were performed as it has been described by Monjo et al. [49]. ALP activity and Coll-1 mRNA levels were assessed after 14 days.

\subsection{Statistical Analysis}

Data were compared by a two way ANOVA in SigmaPlot 11 (Systat Software, San José, CA, USA). A normality test was performed; once this was passed, all samples were compared in pairs using the Holm-Sidak method. ANOVA was performed on ranks when the normality test failed, using the Student-Newman-Keuls test or Dunn's test for pairwise comparison. Significance levels were set to significant $* p \leq 0.05$ and highly significant $* * p \leq 0.01$. All data were displayed as arithmetic mean values with standard deviation when the data were distributed normally and as median values with interquartile range when the data were not distributed normally. The results of the cell study were compared by a paired student's t-test and displayed as box plots of the median values (Q2) with the 5, $25(\mathrm{Q} 1), 75(\mathrm{Q} 3)$, and 95 percentiles.

\section{Conclusions}

Cathodic polarization under acidic conditions can be used to coat commercial implant surfaces with the growth-promoting agent EMD. Moreover, the coated surfaces revealed intact EMD that was bio-available and maintained its function. The hydride layer of the original SBAE surfaces could be activated by the process as an intermediate stage of charged hydride that acted as a coupling layer for the biomolecule. The coating presented in this study can be applied in an integrated manner of a one-step process that does not require modifications or pre-treatments of the commercially available base material prior to coating and is gentle enough to not inactivate or degrade protein amino acid-based biomolecules. Although the current study only assessed Ti and TiZr with SBAE surfaces, the process should be transferable to other metallic materials that develop a hydride layer during cathodic polarization and may potentially be used for other biomolecules that ionize under acidic conditions as well.

\section{Acknowledgments}

This work was supported by the Norwegian Research Council (Grants No. 203034 and 203036) and by the Ministerio de Ciencia e Innovación del Gobierno de España (Torres Quevedo contract to MR, and Ramón y Cajal contract to MM). The study materials, Ti and TiZr coins and EMD, were kindly provided by Institut Straumann AG, Basel, Switzerland. The authors are especially thankful for the excellent technical support and assistance from Martin Fleissner Sunding (Department of Physics, University of Oslo) for the XPS analysis, from Alexander Azarov (Centre for Materials Research, University of Oslo) for the SIMS analysis. 


\section{Author Contributions}

All authors greatly contributed to the development of this manuscript. Matthias J. Frank designed and conducted the experiments, interpreted the results, and was responsible for composing this manuscript. Martin S. Walter was involved in designing and conducting the experiments, contributed largely to the interpretation of the results, and helped refining this manuscript. Marina Rubert and Marta Monjo conducted the in vitro experiments and assisted with the interpretation of the results. Bernd Thiede conducted the MALDI experiments and assisted interpreting the results. Janne E. Reseland assisted with the interpretation of the results and helped refining this manuscript. Håvard J. Haugen and Ståle Petter Lyngstadaas acquired the funding for the research conducted in this paper, were largely involved during the design of the study, helped interpreting the results and assisted refining this manuscript.

\section{Conflicts of Interest}

The authors declare no conflict of interest

\section{References}

1. Albrektsson, T.; Sennerby, L.; Wennerberg, A. State of the art of oral implants. Periodontol 2000 2008, 47, 15-26.

2. Zhang, F.; Yang, G.L.; He, F.M.; Zhang, L.J.; Zhao, S.F. Cell response of titanium implant with a roughened surface containing titanium hydride: An in vitro study. J. Oral Maxillofac. Surg. 2010, $68,1131-1139$.

3. Le Guehennec, L.; Soueidan, A.; Layrolle, P.; Amouriq, Y. Surface treatments of titanium dental implants for rapid osseointegration. Dent. Mater. 2007, 23, 844-854.

4. Boyan, B.D.; Hummert, T.W.; Dean, D.D.; Schwartz, Z. Role of material surfaces in regulating bone and cartilage cell response. Biomaterials 1996, 17, 137-146.

5. Buser, D.; Schenk, R.K.; Steinemann, S.; Fiorellini, J.P.; Fox, C.H.; Stich, H. Influence of surface characteristics on bone integration of titanium implants. A histomorphometric study in miniature pigs. J. Biomed. Mater. Res. 1991, 25, 889-902.

6. Fini, M.; Cigada, A.; Rondelli, G.; Chiesa, R.; Giardino, R.; Giavaresi, G.; Nicoli Aldini, N.; Torricelli, P.; Vicentini, B. In vitro and in vivo behaviour of Ca- and P-enriched anodized titanium. Biomaterials 1999, 20, 1587-1594.

7. Ishizawa, H.; Fujino, M.; Ogino, M. Mechanical and histological investigation of hydrothermally treated and untreated anodic titanium oxide films containing $\mathrm{Ca}$ and P. J. Biomed. Mater. Res. 1995, 29, 1459-1468.

8. Wong, M.; Eulenberger, J.; Schenk, R.; Hunziker, E. Effect of surface topology on the osseointegration of implant materials in trabecular bone. J. Biomed. Mater. Res. 1995, 29, 1567-1575.

9. Lamolle, S.F.; Monjo, M.; Rubert, M.; Haugen, H.J.; Lyngstadaas, S.P.; Ellingsen, J.E. The effect of hydrofluoric acid treatment of titanium surface on nanostructural and chemical changes and the growth of MC3T3-E1 cells. Biomaterials 2009, 30, 736-742. 
10. Frank, M.J.; Walter, M.S.; Lyngstadaas, S.P.; Wintermantel, E.; Haugen, H.J. Hydrogen content in titanium and a titanium-zirconium alloy after acid etching. Mater. Sci. Eng. C Mater. Biol. Appl. 2013, 33, 1282-1288.

11. Rønold, H.J.; Ellingsen, J.E. Effect of micro-roughness produced by $\mathrm{TiO}_{2}$ blasting. Tensile testing of bone attachment by using coin-shaped implants. Biomaterials 2002, 23, 4211-4219.

12. Rønold, H.J.; Lyngstadaas, S.P.; Ellingsen, J.E. A study on the effect of dual blasting with $\mathrm{TiO}_{2}$ on titanium implant surfaces on functional attachment in bone. J. Biomed. Mater. Res. A 2003, 67A, 524-530.

13. Szmukler-Moncler, S.; Bischof, M.; Nedir, R.; Ermrich, M. Titanium hydride and hydrogen concentration in acid-etched commercially pure titanium and titanium alloy implants: A comparative analysis of five implant systems. Clin. Oral Implants Res. 2010, 21, 944-950.

14. Wen, H.B.; Wolke, J.G.; de Wijn, J.R.; Liu, Q.; Cui, F.Z.; de Groot, K. Fast precipitation of calcium phosphate layers on titanium induced by simple chemical treatments. Biomaterials 1997, $18,1471-1478$.

15. Wen, H.B.; Liu, Q.; de Wijn, J.R.; de Groot, K.; Cui, F.Z. Preparation of bioactive microporous titanium surface by a new two-step chemical treatment. J. Mater. Sci. Mater. Med. 1998, 9, 121-128.

16. Khang, W.; Feldman, S.; Hawley, C.E.; Gunsolley, J. A multi-center study comparing dual acid-etched and machined-surfaced implants in various bone qualities. J. Periodontol. 2001, 72, 1384-1390.

17. Conforto, E.; Caillard, D. A fast method for determining favourable orientation relationships and interface planes: Application to titanium-titanium hydrides transformations. Acta Biomater. 2007, 55, 785-798.

18. Perrin, D.; Szmukler-Moncler, S.; Echikou, C.; Pointaire, P.; Bernard, J.P. Bone response to alteration of surface topography and surface composition of sandblasted and acid etched (SLA) implants. Clin. Oral Implants Res. 2002, 13, 465-469.

19. Cheng, Z.; Zhang, F.; He, F.; Zhang, L.; Guo, C.; Zhao, S.; Yang, G. Osseointegration of titanium implants with a roughened surface containing hydride ion in a rabbit model. Oral Surg. Oral Med. Oral Pathol. Oral Radiol. Endod. 2010, 110, e5-e12.

20. Rupp, F.; Scheideler, L.; Olshanska, N.; de Wild, M.; Wieland, M.; Geis-Gerstorfer, J. Enhancing surface free energy and hydrophilicity through chemical modification of microstructured titanium implant surfaces. J. Biomed. Mater. Res. A 2006, 76, 323-334.

21. Zhao, G.; Schwartz, Z.; Wieland, M.; Rupp, F.; Geis-Gerstorfer, J.; Cochran, D.L.; Boyan, B.D. High surface energy enhances cell response to titanium substrate microstructure. J. Biomed. Mater. Res. A 2005, 74, 49-58.

22. Lamolle, S.F.; Monjo, M.; Lyngstadaas, S.P.; Ellingsen, J.E.; Haugen, H.J. Titanium implant surface modification by cathodic reduction in hydrofluoric acid: Surface characterization and in vivo performance. J. Biomed. Mater. Res. A 2009, 88, 581-588.

23. Kang, B.S.; Sul, Y.T.; Oh, S.J.; Lee, H.J.; Albrektsson, T. XPS, AES and SEM analysis of recent dental implants. Acta Biomater. 2009, 5, 2222-2229. 
24. Cooper, L.; Zhou, Y.; Takebe, J.; Guo, J.; Abron, A.; HolmÈn, A.; Ellingsen, J. Fluoride modification effects on osteoblast behavior and bone formation at $\mathrm{TiO}_{2}$ grit-blasted c.p. titanium endosseous implants. Biomaterials 2006, 27, 926-936.

25. Ellingsen, J.E. Pre-treatment of titanium implants with fluoride improves their retention in bone. J. Mater. Sci. Mater. Med. 1995, 6, 749-753.

26. Ellingsen, J.E.; Johansson, C.B.; Wennerberg, A.; Holmen, A. Improved retention and bone-tolmplant contact with fluoride-modified titanium implants. Int. J. Oral Maxillofac. Implants 2004, 19, 659-666.

27. Mihranyan, A.; Forsgren, J.; Strømme, M.; Engqvist, H. Assessing surface area evolution during biomimetic growth of hydroxyapatite coatings. Langmuir 2008, 25, 1292-1295.

28. Forsgren, J.; Brohede, U.; Piskounova, S.; Mihranyan, A.; Larsson, S.; Strømme, M.; Engqvist, H. In vivo evaluation of functionalized biomimetic hydroxyapatite for local delivery of active agents. J. Biomater. Nanobiotechnol. 2011, 2, 149-154.

29. Piskounova, S.; Forsgren, J.; Brohede, U.; Engqvist, H.; Strømme, M. In vitro characterization of bioactive titanium dioxide/hydroxyapatite surfaces functionalized with BMP-2. J. Biomed. Mater. Res. B Appl. Biomater. 2009, 91, 780-787.

30. Forsgren, J.; Brohede, U.; Strømme, M.; Engqvist, H. Co-loading of bisphosphonates and antibiotics to a biomimetic hydroxyapatite coating. Biotechnol. Lett. 2011, 33, 1265-1268.

31. Brohede, U.; Forsgren, J.; Roos, S.; Mihranyan, A.; Engqvist, H.; Strømme, M. Multifunctional implant coatings providing possibilities for fast antibiotics loading with subsequent slow release. J. Mater. Sci. Mater. Med. 2009, 20, 1859-1867.

32. Åberg, J.; Brohede, U.; Mihranyan, A.; Strømme, M.; Engqvist, H. Bisphosphonate incorporation in surgical implant coatings by fast loading and co-precipitation at low drug concentrations. J. Mater. Sci. Mater. Med. 2009, 20, 2053-2061.

33. Morra, M. Biochemical modification of titanium surfaces: Peptides and ECM proteins. Eur. Cells Mater. 2006, 12, 1-15.

34. Yazici, H.; Fong, H.; Wilson, B.; Oren, E.E.; Amos, F.A.; Zhang, H.; Evans, J.S.; Snead, M.L.; Sarikaya, M.; Tamerler, C. Biological response on a titanium implant-grade surface functionalized with modular peptides. Acta Biomater. 2013, 9, 5341-5352.

35. Lyngstadaas, S.P.; Ellingsen, J.E.; Astra Tech, A.B. Medical prosthetic devices and implants having improved biocompatibility. WO Patent: WO/2002/045,764, 2002.

36. Videm, K.; Lamolle, S.; Monjo, M.; Ellingsen, J.E.; Lyngstadaas, S.P.; Haugen, H.J. Hydride formation on titanium surfaces by cathodic polarization. Appl. Surf. Sci. 2008, 255, 3011-3015.

37. Frank, M.J.; Walter, M.S.; Bucko, M.M.; Pamula, E.; Lyngstadaas, S.P.; Haugen, H.J. Polarization of modified titanium and titanium-zirconium creates nano-structures while hydride formation is modulated. Appl. Surf. Sci. 2013, 282, 7-16.

38. Frank, M.J.; Walter, M.S.; Tiainen, H.; Rubert, M.; Monjo, M.; Lyngstadaas, S.P.; Haugen, H.J. Coating of metal implant materials with strontium. Mater. Sci. Mater. Med. 2013, 24, 2537-2548.

39. Walter, M.S.; Frank, M.J.; Rubert, M.; Monjo, M.; Rønold, H.J.; Lyngstadaas, P.; Haugen, H.J. Bioactive implant surface with electrochemically bound doxycycline promotes bone formation markers in vitro and in vivo. Dent. Mater. 2014, 30, 200-214. 
40. Grandin, H.M.; Gemperli, A.C.; Dard, M. Enamel matrix derivative: A review of cellular effects in vitro and a model of molecular arrangement and functioning. Tissue Eng. Part. B Rev. 2012, 18, 181-202.

41. Lyngstadaas, S.P.; Wohlfahrt, J.C.; Brookes, S.J.; Paine, M.L.; Snead, M.L.; Reseland, J.E. Enamel matrix proteins; old molecules for new applications. Orthod. Craniofac. Res. 2009, 12, 243-253.

42. Cattaneo, V.; Rota, C.; Silvestri, M.; Piacentini, C.; Forlino, A.; Gallanti, A.; Rasperini, G.; Cetta, G. Effect of enamel matrix derivative on human periodontal fibroblasts: Proliferation, morphology and root surface colonization. An in vitro study. J. Periodontal Res. 2003, 38, 568-574.

43. Miron, R.J.; Oates, C.J.; Molenberg, A.; Dard, M.; Hamilton, D.W. The effect of enamel matrix proteins on the spreading, proliferation and differentiation of osteoblasts cultured on titanium surfaces. Biomaterials 2010, 31, 449-460.

44. Iqbal, M.K.; Bamaas, N. Effect of enamel matrix derivative EMDOGAIN ${ }^{\circledR}$ upon periodontal healing after replantation of permanent incisors in beagle dogs. Dent. Traumatol. 2001, 17, 36-45.

45. Lagerström-fermér, M.; Nilsson, M.; Bäckman, B.; Salido, E.; Shapiro, L.; Pettersson, U.; Landegren, U. Amelogenin signal peptide mutation: Correlation between mutations in the amelogenin gene (AMGX) and manifestations of X-linked amelogenesis imperfecta. Genomics 1995, 26, 159-162.

46. Iwata, T.; Yamakoshi, Y.; Hu, J.C.; Ishikawa, I.; Bartlett, J.D.; Krebsbach, P.H.; Simmer, J.P. Processing of ameloblastin by MMP-20. J. Dent. Res. 2007, 86, 153-157.

47. Zilm, P.S.; Bartold, P.M. Proteomic identification of proteinase inhibitors in the porcine enamel matrix derivative, $\operatorname{EMD}((\mathrm{R}))$. J. Periodontal Res. 2011, 46, 111-117.

48. Quarles, L.D.; Yohay, D.A.; Lever, L.W.; Caton, R.; Wenstrup, R.J. Distinct proliferative and differentiated stages of murine MC3T3-E1 cells in culture: An in vitro model of osteoblast development. J. Bone Miner. Res. 1992, 7, 683-692.

49. Monjo, M.; Rubert, M.; Ellingsen, J.E.; Lyngstadaas, S.P. Rosuvastatin promotes osteoblast differentiation and regulates SLCO1A1 transporter gene expression in MC3T3-E1 cells. Cell. Physiol. Biochem. 2010, 26, 647-656.

50. Reseland, J.E.; Reppe, S.; Larsen, A.M.; Berner, H.S.; Reinholt, F.P.; Gautvik, K.M.; Slaby, I.; Lyngstadaas, S.P. The effect of enamel matrix derivative on gene expression in osteoblasts. Eur. J. Oral Sci. 2006, 114, 205-211.

51. Rubert, M.; Ramis, J.M.; Vondrasek, J.; Gaya, A.; Lyngstadaas, S.P.; Monjo, M. Synthetic peptides analogue to enamel proteins promote osteogenic differentiation of MC3T3-E1 and mesenchymal stem cells. J. Biomater. Tissue Eng. 2011, 1, 198-209.

52. Mumulidu, A.; Hildebrand, B.; Fabi, B.; Hammarstrom, L.; Cochran, D.L.; Dard, M.; Lemoult, S. Purification and analysis of a $5 \mathrm{kDa}$ component of enamel matrix derivative. J. Chromatogr. B Anal. Technol. Biomed. Life Sci. 2007, 857, 210-218.

53. Fincham, A.G.; Moradian-Oldak, J. Amelogenin post-translational modifications: Carboxy-terminal processing and the phosphorylation of bovine and porcine "trap" and "Irap" amelogenins. Biochem. Biophys. Res. Commun. 1993, 197, 248-255.

54. Riksen, E.A.; Petzold, C.; Brookes, S.; Lyngstadaas, S.P.; Reseland, J.E. Human osteoblastic cells discriminate between 20-kDa amelogenin isoforms. Eur. J. Oral Sci. 2011, 119, 357-365. 
55. Cohen, S.L.; Chait, B.T. Influence of matrix solution conditions on the MALDI-MS analysis of peptides and proteins. Anal. Chem. 1996, 68, 31-37.

56. Morra, M.; Cassinelli, C.; Cascardo, G.; Cahalan, P.; Cahalan, L.; Fini, M.; Giardino, R. Surface engineering of titanium by collagen immobilization. Surface characterization and in vitro and in vivo studies. Biomaterials 2003, 24, 4639-4654.

57. Bernhard, N.; Berner, S.; de Wild, M.; Wieland, M. The binary TiZr alloy-A newly developed Ti alloy for use in dental implants. Forum Implantol. 2009, 5, 30-39.

58. Gestrelius, S.; Andersson, C.; Johansson, A.C.; Persson, E.; Brodin, A.; Rydhag, L.; Hammarstrom, L. Formulation of enamel matrix derivative for surface coating. Kinetics and cell colonization. J. Clin. Periodontol. 1997, 24, 678-684.

59. Gestrelius, S.; Lyngstadaas, S.P.; Hammarström, L. Emdogain-periodontal regeneration based on biomimicry. Clin. Oral Investig. 2000, 4, 120-125.

60. Al-Nawas, B.; Bragger, U.; Meijer, H.J.; Naert, I.; Persson, R.; Perucchi, A.; Quirynen, M.; Raghoebar, G.M.; Reichert, T.E.; Romeo, E.; et al. A double-blind randomized controlled trial (RCT) of titanium-13zirconium versus titanium grade IV small-diameter bone level implants in edentulous mandibles - Results from a 1-year observation period. Clin. Implant Dent. Relat. Res. 2012, 14, 896-904.

61. Gottlow, J.; Dard, M.; Kjellson, F.; Obrecht, M.; Sennerby, L. Evaluation of a new titanium-zirconium dental implant: A biomechanical and histological comparative study in the mini pig. Clin. Implant Dent. Relat. Res. 2010, 24, 538-545.

62. Grandin, H.M.; Berner, S.; Dard, M. A review of titanium zirconium (TiZr) alloys for use in endosseous dental implants. Materials 2012, 5, 1348-1360.

63. Buser, D.; Broggini, N.; Wieland, M.; Schenk, R.K.; Denzer, A.J.; Cochran, D.L.; Hoffmann, B.; Lussi, A.; Steinemann, S.G. Enhanced bone apposition to a chemically modified sla titanium surface. J. Dent. Res. 2004, 83, 529-533.

64. Lu, X.; Wang, Y.; Yang, X.; Zhang, Q.; Zhao, Z.; Weng, L.T.; Leng, Y. Spectroscopic analysis of titanium surface functional groups under various surface modification and their behaviors in vitro and in vivo. J. Biomed. Mater. Res. A 2008, 84, 523-534.

65. Donos, N.; Hamlet, S.; Lang, N.P.; Salvi, G.E.; Huynh-Ba, G.; Bosshardt, D.D.; Ivanovski, S. Gene expression profile of osseointegration of a hydrophilic compared with a hydrophobic microrough implant surface. Clin Oral Implants Res. 2011, 22, 365-372.

66. Moulder, J.F.; Stickle, W.F.; Sobol, P.E.; Bomben, K.D. Handbook of X-ray Photoelectron Spectroscopy; Physical Electronics Inc.: Edan Prairie, MA, USA, 1995.

67. Cai, K.; Bossert, J.; Jandt, K.D. Does the nanometre scale topography of titanium influence protein adsorption and cell proliferation? Colloids Surf. B Biointerfaces 2006, 49, 136-144.

(C) 2014 by the authors; licensee MDPI, Basel, Switzerland. This article is an open access article distributed under the terms and conditions of the Creative Commons Attribution license (http://creativecommons.org/licenses/by/3.0/). 